\title{
Maximizing Non-monotone Submodular Set Functions Subject to Different Constraints: Combined Algorithms
}

\author{
Salman Fadaei* $\quad$ MohammadAmin Fazli ${ }^{\dagger} \quad$ MohammadAli Safari ${ }^{\ddagger}$
}

January 18, 2021

We study the problem of maximizing constrained non-monotone submodular functions and provide approximation algorithms that improve existing algorithms in terms of either the approximation factor or simplicity. Our algorithms combine existing local search and greedy based algorithms. Different constraints that we study are exact cardinality and multiple knapsack constraints. For the multiple-knapsack constraints we achieve a $(0.25-2 \epsilon)$-factor algorithm.

We also show, as our main contribution, how to use the continuous greedy process for non-monotone functions and, as a result, obtain a 0.13-factor approximation algorithm for maximization over any solvable down-monotone polytope. The continuous greedy process has been previously used for maximizing smooth monotone submodular function over a downmonotone polytope [CCPV08. This implies a 0.13-approximation for several discrete problems, such as maximizing a non-negative submodular function subject to a matroid constraint and/or multiple knapsack constraints.

\section{Introduction}

Submodularity is the discrete analogous of convexity. Submodular set functions naturally arise in several different important problems including cuts in graphs [IFF01, GW94, rank functions of matroids [E70, and set covering problems [F98. The problem of maximizing a submodular function is NP-hard as it generalizes many important problems such as Maximum Cut [FG95], Maximum Facility Location [B03, AS99], and the Quadratic Cost Partition Problem with nonnegative edge weights GG05.

Definition 1. A function $f: 2^{X} \rightarrow \mathbb{R}_{+}$is called submodular if and only if $\forall A, B \subseteq X, f(A)+$ $f(B) \geq f(A \cap B)+f(A \cup B)$. An alternative definition is that the marginal values of items should be non-increasing, i.e., $\forall A, B \subseteq X, A \subseteq B \subseteq X$ and $x \in X \backslash B, f_{A}(x) \geq f_{B}(x)$, where $f_{A}(x)=f(A \cup\{x\})-f(A) ; f_{A}(x)$ is called the marginal value of $x$ with respect to $A$.

The Submodular Maximization Problem is a pair $(f, \Delta)$ where $f$ is a submodular function and $\Delta$ is the search domain. Our aim is to find a set $A^{*} \in \Delta$ whose value, $f\left(A^{*}\right)$, is maximum. Our focus is on non-monotone submodular functions, i.e., we do not require that $f(A) \leq f(B)$ for $A \subseteq B \subseteq X$.

Definition 2. A packing polytope is a polytope $P \subseteq[0,1]^{X}$ that is down-monotone: If $x, y \in$ $[0,1]^{X}$ with $x \preceq y$ and $y \in P$, then $x \in P$. A polytope $P$ is solvable if we can maximize linear functions over $P$ in polynomial time [Sch03].

A packing polytope constraint binds the search domain $(\Delta)$ to a packing polytope.

\footnotetext{
${ }^{*}$ Faculty of Engineering, University of Tehran, Tehran, Iran. Email: salman.fadaei@gmail.com

${ }^{\dagger}$ Department of Computer Engineering, Sharif University of Technology, Tehran, Iran. Email: fazli@ce.sharif.edu

${ }^{\ddagger}$ Department of Computer Engineering, Sharif University of Technology, Tehran, Iran. Email: msafari@sharif.edu
} 


\begin{tabular}{|l|c|c|c|c|l|}
\hline Constraint & [LMNS09] & [V09] & [GRST10] & Our Result & Claim \\
\hline \hline Exact Cardinality & 0.15 & 0.25 & 0.17 & 0.25 & Simpler \\
\hline k-Knapsacks & 0.2 & - & - & 0.25 & Better ratio \\
\hline Packing Polytope & - & - & - & 0.13 & New ratio \\
\hline
\end{tabular}

Table 1: Comparison of our results with the existing ones.

Definition 3. For a ground set $X, k$ weight vectors $\left\{w^{i}\right\}_{i=1}^{k}$, and $k$ knapsack capacities $\left\{C_{i}\right\}_{i=1}^{k}$ are given. $A$ set $V \subseteq X$ is called packable if $\sum_{j \in V} w_{j}^{i} \leq C_{i}$, for $i=1, \ldots, k$.

The multiple knapsacks constraint forces us to bind search domain to packable subsets of $X$. In the exact cardinality constraint, we have $\Delta=\{S \subseteq X:|S|=k\}$.

Background The problem of maximizing non-monotone submodular functions, with or without some constraints, has been extensively studied in the literature. In [FMV07] a 0.4-factor approximation algorithm was developed for maximizing unconstrained (non-negative, nonmonotone) submodular functions. The approximation factor was very recently improved to 0.41 by Oveis Gharan et al. OV11].

For the constrained variants, Lee et al. [LMNS09, Vondrák [V09], and Gupta et al. [GRST10] provide the best approximation algorithms. Lee et al. [LMNS09] developed a 0.2-approximation for the problem subject to a constant number of knapsack constraints, followed by a 0.25 approximation for cardinality constraint and a 0.15-approximation for the exact cardinality constraint. The latter two approximation factors were later improved by Vondrák [V09] to 0.309 and 0.25 , respectively. As a new way of tackling these problems, Gupta et al. GRST10. provide greedy algorithms that achieve the approximation factor of 0.17 for a knapsack constraint. Greedy algorithms are more common for maximizing monotone submodular functions.

In a recent work, Vondrák [V08, and Calinescu et al. CCPV08] used the idea of multilinear extension of submodular functions and achieved optimal approximation algorithms for the problem of maximizing a monotone submodular function subject to a matroid.

\subsection{Our Results}

We consider the problem subject to different constraints. Our results are summarized in Table 1 and are compared with existing results. We obtain simple algorithms for the exact cardinality constraint, multiple knapsack constraints, and a new approximation algorithm for the solvable packing polytope constraint.

\subsection{Preliminaries}

In this section, we introduce the concepts and terms that we often use throughout this paper.

Multilinear Extension For a submodular function $f: 2^{X} \rightarrow \mathbb{R}_{+}$, the multilinear extension of $f$ is defined as follows [CCPV07]: $F:[0,1]^{X} \rightarrow \mathbb{R}_{+}$and

$$
F(x)=\mathbf{E}[f(x)]=\sum_{S \subseteq X} f(S) \prod_{i \in S} x_{i} \prod_{i \in X \backslash S}\left(1-x_{i}\right) .
$$

This concept is frequently used in recent works [CCPV07, CCPV08, KST09, LMNS09, V09]. The multilinear extension of every submodular function is a smooth submodular function CCPV08. The gradient of $F$ is defined as $\nabla F=\left(\frac{\partial F}{\partial x_{1}}, \ldots, \frac{\partial F}{\partial x_{n}}\right)$.

Matroid A matroid is a pair $\mathcal{M}=(X, \mathcal{I})$ where $\mathcal{I} \subseteq 2^{X}$ and

- $\forall B \in \mathcal{I}, A \subset B \Rightarrow A \in \mathcal{I}$.

- $\forall A, B \in \mathcal{I},|A|<|B| \Rightarrow \exists x \in B \backslash A ; A \cup\{x\} \in \mathcal{I}$. 
Matroid Polytopes A matroid polytope is a solvable packing polytope with special properties. Given a matroid $\mathcal{M}=(X, \mathcal{I})$, we define the matroid polytope as

$$
P(\mathcal{M})=\left\{x \geq 0: \forall S \subseteq X ; \sum_{j \in S} x_{j} \leq r_{\mathcal{M}}(S)\right\}
$$

where $r_{\mathcal{M}}(S)=\max \{|I|: I \subseteq S ; I \in \mathcal{I}\}$ is the rank function of matroid $\mathcal{M}$. This definition shows that the matroid polytope is a packing polytope.

Randomized Pipage Rounding Given a matroid $\mathcal{M}=(X, \mathcal{I})$, the randomized pipage rounding converts a fractional point in the matroid polytope, $y \in P(\mathcal{M})$ into a random set $B \in \mathcal{I}$ such that $\mathbf{E}[f(B)] \geq F(y)$, where $F$ is the multilinear extension of the submodular function $f$ CCPV07, CCPV08, V09].

\subsection{Recent Developments}

There has been some very recent relevant works independent and concurrent to our work. Kulik et al. give an $(0.25-\epsilon)$-approximation algorithm for maximizing non-monotone submodular functions subject to multiple knapsacks [KST11. Chekuri et al. [CVZ11] show that, by using a fractional local search, a 0.325-approximation could be achieved for maximizing non-monotone submodular functions subject to any solvable packing polytope. However, our 0.13-factor approximation algorithm is still of independent interest in that it uses the continuous greedy approach rather than local search and, thus, it would be more efficient in practice.

\section{Exact Cardinality Constraint}

In this section, we propose very simple algorithm for the exact cardinality constraint problem whose approximation factor matches the best existing one, yet it is much simpler and easy to implement. Our algorithm is a simple combination of existing local search or greedy based algorithms. Our main tool is the following useful lemma from Gupta et al. [GRST10].

Lemma 1. (GRST10]) Given sets $C, S_{1} \subseteq X$, let $C^{\prime}=C \backslash S_{1}$ and $S_{2} \subseteq X \backslash S_{1}$. Then, $f\left(S_{1} \cup C\right)+f\left(S_{1} \cap C\right)+f\left(S_{2} \cup C^{\prime}\right) \geq f(C)$.

Let $k$ be the right-hand side of the cardinality constraint.

Theorem 1. There is a 0.25 -factor approximation algorithm for maximizing a non-monotone submodular function subject to an exact cardinality constraint.

Proof. First, we use the local search algorithm of LMNS09 and compute a set $S_{1}$ whose size is $k$ and $2 f\left(S_{1}\right) \geq f\left(S_{1} \cup C\right)+f\left(S_{1} \cap C\right)$ for any $C$ with $|C|=\left|S_{1}\right|=k$. Next, we use the greedy algorithm of [GRST10] and compute a set $S_{2} \subseteq X \backslash S_{1}$ of size $k$ such that for any $C^{\prime}$ with $\left|C^{\prime}\right| \leq k, f\left(S_{2}\right) \geq 0.5 f\left(S_{2} \cup C^{\prime}\right)$. Let $C$ be the true optimum and $C^{\prime}=C \backslash S_{1}$. Therefore,

$$
2 f\left(S_{1}\right)+2 f\left(S_{2}\right) \geq f\left(S_{1} \cup C\right)+f\left(S_{1} \cap C\right)+f\left(S_{2} \cup C^{\prime}\right) \geq f(C)=O P T
$$

Thus, the better of $S_{1}$ and $S_{2}$ gives an approximation factor 0.25 .

Here, we have assumed that $k \leq \frac{|X|}{2}$. If not, we can alternatively solve the problem for the derived submodular function $g(S)=f(X \backslash S)$ subject to cardinality constraint $k^{\prime}=|X|-k$.

The approximation factor 0.25 matches that of [V09], though our algorithm is simpler and straightforward to implement. 


\section{Multiple Knapsack Constraints}

Lee et al. LMNS09 propose a 0.2-factor approximation algorithm for the problem. They basically divide the elements into two sets of heavy and light objects and then solve the problem separately for each set and return the maximum of the two solutions.

We improve their result by considering both heavy and light elements together. Our algorithm finds a fractional solution and then integrates it by using independent rounding. We use some of the properties of the independent rounding; For the sake of completeness, we mention it before presenting the main algorithm.

Let $x=\left(x_{1}, \ldots, x_{n}\right)$ be a fractional solution and $\left(X_{1}, \ldots, X_{n}\right) \in\{0,1\}^{n}$ be an integral solution obtained from $x$ by randomized independent rounding. We observe that $\mathbf{E}\left[X_{i}\right]=x_{i}$ and for any subset $T, \mathbf{E}\left[\prod_{i \in T} X_{i}\right]=\prod_{i \in T} x_{i}$, and $\mathbf{E}\left[\prod_{i \in T}\left(1-X_{i}\right)\right]=\prod_{i \in T}\left(1-x_{i}\right)$. Considering these properties, as in [CVZ10] (Theorem II.1) and [GKPS06] (Theorem 3.1), we obtain the following Chernoff-type concentration bound for linear functions of $X_{1}, \ldots, X_{n}$.

Lemma 2. Let $a_{i} \in[0,1]$ and $X=\sum a_{i} X_{i}$ where $\left(X_{1}, \ldots, X_{n}\right)$ are obtained by randomized independent rounding from a starting point $\left(x_{1}, \ldots, x_{n}\right)$. Then (i) for $\delta \in[0,1]$, and $\mu \geq \boldsymbol{E}[X]=$ $\sum a_{i} x_{i}$, we have $\operatorname{Pr}[X \geq(1+\delta) \mu] \leq e^{-\mu \delta^{2} / 3}$ and (ii) for $\delta \geq 1, \operatorname{Pr}[X \geq(1+\delta) \mu] \leq e^{-\mu \delta / 3}$.

The following simple observation will be useful in the presentation of the algorithm.

Lemma 3. Let $f: 2^{X} \rightarrow \mathbb{R}_{+}$be a nonnegative submodular function over $X, A \subseteq X$ and $X^{\prime}=X \backslash A$, then $h: 2^{X^{\prime}} \rightarrow \mathbb{R}_{+}$where $h(S)=f(A \cup S), \forall S \subseteq X^{\prime}$ is a nonnegative submodular function.

Proof. Fix an arbitrary $A \subseteq X$ and define $X^{\prime}=X \backslash A$. Let $S$ and $T$ with $S \subseteq T$ be two arbitrary subsets of $X^{\prime}$. Let $x \in X^{\prime} \backslash T$. To show submodularity of $h$, we need show that $h_{S}(x) \geq h_{T}(x)$. Recalling the definition of $h$, it is equivalent to show $f(S \cup A \cup\{x\})-f(S \cup A) \geq$ $f(T \cup A \cup\{x\})-f(T \cup A)$. But, this is true since $S \cup A \subseteq T \cup A$ and $f$ is submodular. The nonnegativity of $h$ is clear, the desired conclusion.

Our algorithm (Algorithm 1 below) is based on the algorithm of Chekuri et al. [CVZ10. for maximizing monotone submodular functions subject to one matroid and multiple knapsack constraints. We have made some modifications to use it for non-monotone functions.

Input: Elements weights $\left\{c_{i, j}\right\}$, parameter $0<\epsilon<1 /\left(4 k^{2}\right)$, and a non-monotone submodular function $f$

$D \leftarrow \emptyset$.

foreach subset $A$ of at most $1 / \epsilon^{4}$ elements do

0 . Set $D \leftarrow A$ if $f(A)>f(D)$;

1. Redefine $C_{j}=1-\sum_{i \in A} c_{i j}$ for $1 \leq j \leq k$;

2. Let $B$ be the set of items $i \notin A$ such that either $f_{A}(i)>\epsilon^{4} f(A)$ or $c_{i j}>k \epsilon^{3} C j$ for some $j$;

3. Let $x^{*}$ be the fractional solution of the following problem:

$$
\max \left\{H(x): x \in[0,1]^{X^{\prime}} ; \forall j \sum c_{i j} x_{i} \leq(1-\epsilon) C_{j}\right\}
$$

where $X^{\prime}=X \backslash(A \cup B)$, and $H(x)$ is the multilinear extension of $h(S)=f(A \cup S)$, $\forall S \subseteq X^{\prime}$

4. Let $R$ be the result of the independent rounding applied to $x^{*}$;

If $\exists j: \sum_{i} c_{i j} x_{i}>C_{j}$ then $S \leftarrow \emptyset$ else $S \leftarrow R$; Set $D \leftarrow A \cup S$ if $f(A \cup S)>f(D)$;

end

Return $D$.

Algorithm 1: Non-Monotone Maximization Subject to Multiple Knapsacks

The following theorem shows how good our algorithm is. 
Theorem 2. Algorithm 1 returns a solution of expected value at least $(0.25-2 \epsilon)$ OPT.

Proof. The proof follows the line of proofs of [CVZ10] with major changes to adapt it for nonmonotone case. Let $O$ be the optimal solution and $O P T=f(O)$. Assume $|O| \geq \frac{1}{\epsilon^{4}}$; otherwise, our algorithm finds the optimal solution in Line 0 . Sort the elements of $O$ by their decreasing marginal values, and let $A \subseteq O$ be the first $\frac{1}{\epsilon^{4}}$ elements. Consider the iteration in which this set $A$ is chosen. Since $A$ has $\frac{1}{\epsilon^{4}}$ elements, the marginal value of its last element and every element not in $A$ is at most $\epsilon^{4} f(A) \leq \epsilon^{4} O P T$. Hence, throwing away elements whose marginal value is bigger than $\epsilon^{4} f(A)$ does not affect items in $O \backslash A$. We also throw away the set $B \subseteq X \backslash A$ of items whose size in some knapsack is more than $k \epsilon^{3} C j$. In $O \backslash A$, there can be at most $1 /\left(k \epsilon^{3}\right)$ such items for each knapsack, i.e., $1 / \epsilon^{3}$ items in total. Since their marginal values with respect to $A$ and consequently w.r.t. $A \cup O^{\prime}=O \backslash B$ are bounded by $\epsilon^{4} O P T$ (where $O^{\prime}=O \backslash(A \cup B)$ ), by submodularity these items together have marginal value $f(O)-f(O \backslash B) \leq \epsilon O P T$, therefore $f(O \backslash B) \geq(1-\epsilon) O P T$. For set $O^{\prime}$ we have:

$$
h\left(O^{\prime}\right)=f\left(A \cup O^{\prime}\right)=f(O \backslash B) \geq(1-\epsilon) O P T .
$$

The indicator vector $(1-\epsilon) 1_{O^{\prime}}$ is a feasible solution for Problem 1 (specified at step 3 of Algorithm (1). By the concavity of $H(x)$ along the line from the origin to $1_{O^{\prime}}$, we have $H\left((1-\epsilon) 1_{O^{\prime}}\right) \geq(1-\epsilon) h\left(O^{\prime}\right) \geq(1-2 \epsilon) O P T$. By Theorem 4 of [LMNS09] we can compute in polynomial time a fractional solution $x^{*}$ with value:

$$
H\left(x^{*}\right) \geq \frac{1}{4} H\left((1-\epsilon) 1_{O^{\prime}}\right) \geq\left(\frac{1}{4}-\epsilon\right) O P T .
$$

Notice, according to Lemma 3, $h$ is a nonnegative submodular function and we can apply the algorithm of [LMNS09]. Finally, we apply independent rounding to $x^{*}$ and call the resulting set $R$. By the construction of independent rounding, we have $\mathbf{E}[h(R)]=H\left(x^{*}\right)$. However, $R$ might violate some of the knapsack constraints.

Define $P(l)=\left\{x \in[0,1]^{X^{\prime}} ; \forall j \sum c_{i j} x_{i} \leq l C_{j}\right\}$ for $l \geq 1$ and $l$ is integer. Define $A_{1}$ as the event that $\mathbf{1}_{R} \in P(1)$. By definition of $S$, we have $\mathbf{E}[h(S)]=\mathbf{E}\left[h(R) \mid A_{1}\right] \operatorname{Pr}\left[A_{1}\right]$. Analogously, define disjoint events $A_{l}$ such that $\mathbf{1}_{R} \in P(l) \backslash P(l-1)$ for $l \geq 2$ and $l$ is integer.

We have

$$
H\left(x^{*}\right)=\mathbf{E}[h(R)]=\sum_{l \geq 1} \mathbf{E}\left[h(R) \mid A_{l}\right] \operatorname{Pr}\left[A_{l}\right] .
$$

Consider a fixed knapsack constraint $j$. Our fractional solution $x^{*}$ satisfies $\sum c_{i j} x_{i}^{*} \leq$ $(1-\epsilon) C_{j}$. Also, we know that all sizes in the reduced instance are bounded by $c_{i j} \leq k \epsilon^{3} C_{j}$. By scaling, $c_{i j}^{\prime}=c_{i j} /\left(k \epsilon^{3} C_{j}\right)$, we use Lemma $2(i)$ with $\mu=(1-\epsilon) /\left(k \epsilon^{3}\right)$ :

$$
\operatorname{Pr}\left[\sum_{i \in R} c_{i j}>C_{j}\right] \leq \operatorname{Pr}\left[\sum_{i \in R} c_{i j}^{\prime}>(1+\epsilon) \mu\right] \leq e^{-\mu \epsilon^{2} / 3}<e^{-1 / 4 k \epsilon} .
$$

From this, $\operatorname{Pr}\left[A_{1}\right] \geq 1-k e^{-1 / 4 k \epsilon}$ and $\operatorname{Pr}\left[A_{2}\right] \leq k e^{-1 / 4 k \epsilon}$.

Similarly, we calculate the probability of events $A_{l}, l \geq 3$. Let $\delta=(l-2+\epsilon) /(1-\epsilon)$. From Lemma 2 (ii) and using $\mu=(1-\epsilon) /\left(k \epsilon^{3}\right)$,

$$
\operatorname{Pr}\left[\sum_{i \in R} c_{i j}>(l-1) C_{j}\right] \leq \operatorname{Pr}\left[\sum_{i \in R} c_{i j}^{\prime}>(1+\delta) \mu\right] \leq e^{-\mu \delta / 3} \leq e^{-(l-2) /\left(k \epsilon^{3}\right)} \leq e^{-l /\left(3 k \epsilon^{3}\right)} .
$$

Using the union bound, we can write for any $l \geq 3$

$$
\operatorname{Pr}\left[A_{l}\right] \leq \operatorname{Pr}\left[\exists j ; \sum_{i \in R} c_{i j}>(l-1) C_{j}\right] \leq k e^{-l /\left(3 k \epsilon^{3}\right)} .
$$

Since $H$ is concave along rays through the origin, for all $l \geq 3$ we obtain 


$$
\begin{aligned}
\mathbf{E}\left[h(R) \mid A_{l}\right] & \leq \max \{H(x) \mid x \in P(l)\} \\
& \leq 4 l H\left(x^{*}\right) .
\end{aligned}
$$

Plugging our bounds into 2 we obtain

$$
\begin{aligned}
H\left(x^{*}\right) & =\mathbf{E}\left[h(R) \mid A_{1}\right] \operatorname{Pr}\left[A_{1}\right]+\sum_{l \geq 2} \mathbf{E}\left[h(R) \mid A_{l}\right] \operatorname{Pr}\left[A_{l}\right] \\
& \leq \mathbf{E}\left[h(R) \mid A_{1}\right] \operatorname{Pr}\left[A_{1}\right]+8 k H\left(x^{*}\right) e^{-1 /(4 k \epsilon)}+4 k H\left(x^{*}\right) \sum_{l \geq 3} l e^{-l /\left(3 k \epsilon^{3}\right)} \\
& =\mathbf{E}[h(S)]+4 k H\left(x^{*}\right)\left(2 e^{-1 /(4 k \epsilon)}+\sum_{l \geq 3} l e^{-l /\left(3 k \epsilon^{3}\right)}\right) .
\end{aligned}
$$

Let $q=e^{-1 /\left(3 k \epsilon^{3}\right)}$. Using formula $\sum_{l \geq 3} l q^{l}=\frac{3 q^{3}}{1-q}+\frac{q^{4}}{(1-q)^{2}} \leq q^{3 \epsilon^{2}}$, we obtain $\sum_{l \geq 3} l e^{-l /\left(3 k \epsilon^{3}\right)} \leq$ $e^{-1 /(k \epsilon)}$.

Therefore, we obtain $H\left(x^{*}\right) \leq \mathbf{E}[h(S)]+4 k H\left(x^{*}\right) e^{-1 / k \epsilon}$. Using $4 k e^{-1 / k \epsilon}<\epsilon$ for $\epsilon<$ $\min \left\{1 / k^{2}, 0.001\right\}$, we get $\mathbf{E}[h(S)] \geq(1-\epsilon) H\left(x^{*}\right)$. Therefore, we have a feasible solution of expected value $\mathbf{E}[f(S \cup A)] \geq(1-\epsilon) H\left(x^{*}\right) \geq(1 / 4-2 \epsilon) O P T$.

\section{Packing Polytope Constraint}

In this section, we adapt the continuous greedy process for non-monotone submodular functions and propose an algorithm for solving the optimization problems subject to a packing polytope constraint. As an application of the technique, we then consider the problem of submodular maximization subject to both one matroid and multiple knapsacks constraints. Finally, we briefly show how to replace this continuous process with a polynomial time discrete process without suffering much.

\subsection{Continuous greedy process for non-monotone functions}

Similar to CCPV08, the greedy process starts with $y(0)=\mathbf{0}$ and increases over a unit time interval as follows:

$$
\frac{d y}{d t}=v_{\max }(y)
$$

where $v_{\max }(y)=\operatorname{argmax}_{v \in P}(v \cdot \nabla F(y))$. When $F$ is a non-monotone smooth submodular function, we have

Lemma 4. $y(1) \in P$ and $F(y(1)) \geq\left(1-e^{-1}\right)\left(F(x \vee y(1))-F_{D M A X}\right)$, where $x \in P$, and $F_{D M A X}=\max _{0 \leq t \leq 1} F(y(1)-y(t))$.

Proof. The proof is essentially similar to that of [CPV08 with some modifications to adapt it for non-monotone functions. First, the trajectory for $t \in[0,1]$ is contained in $\mathrm{P}$ since

$$
y(t)=\int_{0}^{t} v_{\max }(y(\tau)) d \tau
$$

is a convex linear combination of vectors in $P$. To prove the approximation guarantee, fix a point $y$. Consider a direction $v^{*}=(x \vee y)-y=(x-y) \vee 0$. This is a non-negative vector; since $v^{*} \leq x \in P$ and $P$ is down-monotone, we also have $v^{*} \in P$. Consider the ray of direction $v^{*}$ starting at $y$, and the function $F\left(y+\xi v^{*}\right), \xi \geq 0$. The directional derivative of $F$ along this ray is $\frac{d F}{d \xi}=v^{*} . \nabla F$. Since $F$ is smooth submodular (that means, each entry $\frac{\partial F}{\partial y_{j}}$ of $\nabla F$ is nonincreasing with respect to $\left.y_{j}\right)$ and $v^{*}$ is nonnegative, $\frac{d F}{d \xi}$ is non-increasing too and $F\left(y+\xi v^{*}\right)$ is concave in $\xi$. By concavity, we have

$$
F\left(y(1)+v^{*}\right)-F(y(t)) \leq F\left(y(t)+v^{*}\right)-F(y(t))+F(y(1)-y(t)) \leq v^{*} . \nabla F(y(t))+F_{D M A X} .
$$

Since $v^{*} \in P$ and $v_{\max }(y) \in P$ maximizes $v \cdot \nabla F(y)$ over all vectors $v \in P$, we get

$$
v_{\max }(y) . \nabla F(y) \geq v^{*} . \nabla F(y) \geq F\left(y(1)+v^{*}\right)-F_{D M A X}-F(y) .
$$


We now get back to the continuous process and analyze $F(y(t))$. Using the chain rule and the inequality (3), we get

$$
\frac{d F}{d t}=\sum_{j} \frac{\partial F}{\partial y_{j}} \frac{d y_{j}}{d t}=v_{\max }(y(t)) . \nabla F(y(t)) \geq F(x \vee y(1))-F_{D M A X}-F(y(t)) .
$$

Thus, $F(y(t))$ dominates the solution of the differential equation

$$
\frac{d \phi}{d t}=F(x \vee y(1))-F_{D M A X}-\phi(t)
$$

which means $\phi(t)=\left(1-e^{-t}\right)\left(F(x \vee y(1))-F_{D M A X}\right)$. Therefore, $F(y(t)) \geq\left(1-e^{-t}\right)(F(x \vee$ $\left.y(1))-F_{D M A X}\right)$.

\subsection{Extending Smooth Local Search.}

As our final tool for obtaining the main algorithm of this section, we propose an algorithm for the following problem: Let $f$ be a sumbodular function and $F$ be its multilinear extension. Let $u_{i} \in[0,1], 1 \leq i \leq n$, be a set of upper bound variables and $\mathcal{U}:=\left\{0 \leq y_{i} \leq u_{i} \quad \forall i \in X\right\}$. We want to maximize $F$ over the region $\mathcal{U}$ :

$$
\max \{F(y): y \in \mathcal{U}\}
$$

For this, we extend the 0.4-approximation algorithm (Smooth Local Search or SLS) of FMV07 as follows. We call our algorithm $F M V_{Y}$.

We define a discrete set $\zeta$ of values in $[0,1]$, where $\zeta=\{p . \delta: 0 \leq p \leq 1 / \delta\}, \delta=\frac{1}{8 n^{4}}$ and $p$ is integer. The algorithm returns a vector whose values come from the discrete set $\zeta$. We show that such a discretization does not substantially harm our solution, yet it reduces the running time.

Let $U$ be a multiset containing $s_{i}=\left\lfloor\frac{1}{\delta} u_{i}\right\rfloor$ copies of each element $i \in X$. We define a set function $g: 2^{U} \rightarrow \mathbb{R}_{+}$with $g(T)=F\left(\ldots, \frac{\left|T_{i}\right|}{s_{i}}, \ldots\right)$, where $T \subseteq U$ and $T_{i}$ contains all copies of $i$ in $T$. The function $g$ has been previously introduced in [LMNS09] and proved to be submodular. Let $B$ be the solution of running the SLS algorithms for maximizing $g$ and $y$ be its corresponding vector.

Based on [FMV07, we have $g(B) \geq 0.4 g(A), \quad \forall A \in U$; thus

$$
F(y) \geq 0.4 F(z), \quad \forall z \in \mathcal{U} \cap \zeta^{n} .
$$

and we can prove the following claim.

Claim 1. For any $x \in \mathcal{U}, 2.5 F(y) \geq F(x)-\frac{f_{\max }}{4 n^{2}}$, where $f_{\max }=\max \{f(i): i \in X\}$.

Proof. Let $z$ be the point in $\zeta^{n} \cap \mathcal{U}$ that minimizes $\sum_{i=1}^{n}\left(x_{i}-z_{i}\right)$. By Claim 3 of [LMNS09, $F(z) \geq F(x)-\frac{f_{\max }}{4 n^{2}}$. Using the inequality (4), we get $F(y) \geq 0.4\left(F(x)-\frac{f_{\max }}{4 n^{2}}\right)$. This completes the proof. 


\subsection{The Algorithm}

We now present our algorithm for maximizing a smooth submodular function over a solvable packing polytope:

Input: A packing polytope $P$ and a smooth submodular function $F$

1. $y_{1} \longleftarrow$ The result of running the continuous greedy process.

2. $y_{1}^{\prime} \longleftarrow \operatorname{argmax}_{0 \leq t \leq 1} F\left(y_{1}-y(t)\right)$.

3. $y_{1 \max } \longleftarrow$ The result of running $F M V_{Y}$ with the upper bound $y_{1}$.

4. $y_{2} \longleftarrow$ The result of running the greedy process over the new polytope $P^{\prime}$ which is

built by adding constraints $y_{i} \leq 1-y_{1_{i}}$ for any $1 \leq i \leq n$ to $P$. Note that $P^{\prime}$ is a

down-monotone polytope.

5. $y_{2}^{\prime} \longleftarrow \operatorname{argmax}_{0 \leq t \leq 1} F\left(y_{2}-y(t)\right)$.

6. Return $\operatorname{argmax}\left(F\left(y_{1}\right), F\left(y_{2}\right), F\left(y_{1 \max }\right), F\left(y_{1}^{\prime}\right), F\left(y_{2}^{\prime}\right)\right)$.

Algorithm 2: Continuous greedy process for non-monotone functions

Theorem 3. The above algorithm is a $\frac{2 e-2}{13 e-9}$-approximation algorithm for the problem of maximizing a smooth submodular function $F$ over a solvable packing polytope $P$.

Proof. Suppose $x^{*} \in P$ is the optimum and $F\left(x^{*}\right)=O P T$. By Lemma 4, $F\left(y_{1}\right) \geq(1-$ $\left.e^{-1}\right)\left(F\left(x^{*} \vee y_{1}\right)-F\left(y_{1}^{\prime}\right)\right)$. We also have $F\left(y_{2}\right) \geq\left(1-e^{-1}\right)\left(F\left(x^{\prime} \vee y_{2}\right)-F\left(y_{2}^{\prime}\right)\right)$, where $x^{\prime}=$ $x^{*}-\left(x^{*} \wedge y_{1}\right)$. Note that $x^{\prime} \in P^{\prime}$. By Claim 1, we also have $F\left(y_{1 \max }\right) \geq 0.4\left(F\left(x^{*} \wedge y_{1}\right)-\frac{f_{\max }}{4 n^{2}}\right)$ as $x^{*} \wedge y_{1} \preceq y_{1}$.

By adding up the above inequalities, we get

$$
\begin{aligned}
& \frac{e}{e-1}\left(F\left(y_{1}\right)+F\left(y_{2}\right)\right)+F\left(y_{1}^{\prime}\right)+F\left(y_{2}^{\prime}\right)+2.5 F\left(y_{1 \max }\right) \\
& \geq F\left(x^{*} \vee y_{1}\right)+F\left(x^{\prime} \vee y_{2}\right)+F\left(x^{*} \wedge y_{1}\right)-\frac{f_{\max }}{4 n^{2}} \\
& \geq F\left(x^{*}\right)-\frac{f_{\max }}{4 n^{2}}=O P T-\frac{f_{\max }}{4 n^{2}} .
\end{aligned}
$$

Therefore, the approximation factor of the algorithm is at least $\frac{2 e-2}{13 e-9} O P T$.

Both one matroid and multiple knapsacks As a direct result of the above theorem, we propose the first approximation algorithm for maximizing a submodular function subject to both one matroid and multiple knapsacks. This problem was solved (approximately) in [CV09] for monotone submodular functions.

Theorem 4. There exists an algorithm with expected value of at least $\left(\frac{2 e-2}{13 e-9}-3 \epsilon\right) O P T$ for the problem of maximizing any non-monotone submodular function subject to one matroid and multiple knapsacks.

Proof. The intersection of the polytopes corresponding to one matroid and multiple knapsacks is still a solvable packing polytope. Thus, we can achieve a fractional solution by using Algorithm 2 together with the enumeration phase (as in Algorithm 1), and then we can round the fractional solution into the integral one using randomized pipage rounding.

Our algorithm is very similar to that of [CV09] with some modifications to adapt it for nonmonotone functions. As the two algorithms are similar, we only highlight the modifications to our algorithm.

The algorithm in [CV09] is for maximizing monotone submodular functions subject to one matroid and multiple knapsacks and uses partial enumeration. At each iteration, after getting rid of all items of large value or size, it defines an optimization problem with scaled down constraints. Since the objective function is monotone, the reduced problem at each iteration is solved using continuous greedy algorithm to find a fractional solution within a factor $1-1 / e$ of the optimal.

For our case, we cannot use the continuous greedy algorithm as our function is not monotone. Instead, we use Algorithm 2 to solve the reduced problem and achieve a fractional solution with approximation factor $\frac{2 e-2}{13 e-9}$. The final step of the two algorithms are identical. At each iteration, 
we apply randomized pipage rounding to the fractional solution with respect to contracted matroid of the that iteration. The result is the set with maximum objective functions over all iterations.

Our analysis is similar to that of [CV09] except that our approximation factor for the reduced problem (at each iteration) is $\frac{2 e-2}{13 e-9}$ as opposed to $1-1 / e$ of [CV09]. So, the same analysis works with the two approximation factors exchanged.

Note that, because of considerations in the design of the algorithm, randomized pipage rounding does not violate, with high probability, the capacity constraints on knapsacks and, therefore, our solution is a feasible one with constant probability. We remark that the argument for the concentration bound in CV09] is applicable to our analysis, as well.

\subsection{Discretizing Continuous Process}

In order to obtain a polynomial time, we discretize the continuous greedy process for nonmonotone functions and show that by taking small enough time steps, this process only introduces a small error that is negligible and the solution to the differential inequality does not significantly change.

Let $\delta=\frac{1}{n^{2}}$, and $\zeta=\{p . \delta: 0 \leq p \leq 1 / \delta\}$ be a set of discrete values. We set the unit time interval equal to $\delta$ in Algorithm 2, and change lines 2 and 5 of it as follows.

$$
\begin{array}{ll}
2 & y_{1}^{\prime} \longleftarrow \operatorname{argmax} F\left(y_{1}-y(t)\right), \forall t \in \zeta \\
5 & y_{2}^{\prime} \longleftarrow \operatorname{argmax} F\left(y_{2}-y(t)\right), \forall t \in \zeta
\end{array}
$$

and obtain the following lemma which is weaker (but not very different) than Lemma 4.

Lemma 5. $y(1), y_{1}^{\prime} \in P$ and $F(y(1)) \geq\left(1-e^{-1}\right)\left(F(x \vee y(1))-F\left(y_{1}^{\prime}\right)\right)-o(1) O P T$, where $x \in P$, where $P$ is any solvable packing polytope.

\section{Acknowledgement}

The Authors are grateful to Vahab Mirrokni for his help in all the steps of the preparation of this paper. The first author is also thankful to Ali Moeini (his M.Sc. advisor), Dara Moazzami, and Jasem Fadaei for their help and advice. The authors also would like to acknowledge the anonymous referees for the their useful comments and suggestions.

\section{References}

[AS99] A. A. Ageev and M. I. Sviridenko, An 0.828-approximation algorithm for the uncapacitated facility location problem. Discrete Appl. Math., 93, 149-156, 1999.

[AS04] A. Ageev and M. Sviridenko. Pipage rounding: a new method of constructing algorithms with proven performance guarantee. J. of Combinatorial Optimization, 8:307-328, 2004.

[B03] S. Benati. An improved Branch \& Bound method for the uncapacitated competitive location problem. Annals of Operations Research 122,no. 1, 43-58, 2003.

[CCPV07] G. Calinescu, C. Chekuri, M. Pál and J. Vondrák. Maximizing a monotone submodular function under a matroid constraint. Proc. of 12th IPCO, 182-196, 2007.

[CCPV08] G. Calinescu, C. Chekuri, M. Pál and J. Vondrák. Maximizing a submodular set function subject to a matroid constraint. To appear in SIAM Journal on Computing, special issue for STOC 2008.

[CKP00] A. Caprara, H. Kellerer and U. Pferschy. Approximation Algorithms for Knapsack Problems with Cardinality Constraints. European J. Oper. Res. 123, 333-345, 2000. 
[CV09] C. Chekuri, J. Vondrák. Randomized Pipage Rounding for Matroid Polytopes and Applications. arXiv:0909.4348v1, 2009.

[CVZ10] C. Chekuri, J. Vondrák, R. Zenklusen. Dependent randomized rounding via exchange properties of combinatorial structures. Proc. of IEEE FOCS, 2010. A more detailed version available at arXiv:0909.4348v2.

[CVZ11] C. Chekuri, J. Vondrák, and R. Zenklusen. Submodular function maximization via the multilinear relaxation and contention resolution schemes. to appear in STOC 2011.

[Dug09] S. Dughmi. Submodular Functions: Extensions, Distributions, and Algorithms A Survey. arXiv:0912.0322v3, 2009.

[E70] J. Edmonds. Matroids, submodular functions, and certain polyhedra. Journal of Combinatorial Structures and Their Applications, 69-87, 1970.

[F98] U. Feige. A threshold of $\ln \mathrm{n}$ for approximating set cover. Journal of the ACM, 45(4):634-652, 1998.

[FG95] U. Feige, and M.X. Goemans. Approximating the value of two prover proof systems, with applications to MAX 2SAT and MAX DICUT Proceedings of the Third Israel Symposium on Theory of Computing and Systems 388, 1995.

[FMV07] U. Feige, V.S.Mirrokni, and J. Vondrák. Maximizing non-monotone submodular functions. In FOCS, 2007.

[GJ79] M. R. Garey and D. S. Johnson. Computers and Intractability: A Guide to the Theory of NP-Completeness. W. H. Freeman and Co., 1979.

[GG05] B. Goldengorin and D. Ghosh. The multilevel search algorithm for the maximization of submodular functions applied to the quadratic cost partition problem. Journal of Global Optimization 32 (1), 65-82, 2005.

[GKPS06] R. Gandhi, S. Khuller, S. Parthasarathy and A. Srinivasan. Dependent rounding and its applications to approximation algorithms. Journal of the ACM 53:324-360, 2006.

[GRST10] A. Gupta, A. Roth, G. Schoenebeck, K. Talwar. Constrained Non-Monotone Submodular Maximization: Offline and Secretary Algorithms, 2010.

[GW94] M. Goemans and P. Williamson. Improved Approximation Algorithms for Maximum Cut and Satisfiability Problems Using Semidefinite Programming. Journal of the ACM (JACM) 42(4), 1115-1145, 1994.

[HMS08] J. Hartline, V. Mirrokni, M. Sundararajan. Optimal marketing strategies over social networks. Proceeding of the 17th international conference on World Wide Web, 189-198, 2008.

[IFF01] S. Iwata, L. Fleischer, and S. Fujishige. A combinatorial strongly polynomial algorithm for minimizing submodular functions Journal of the ACM (JACM) 48(4), 777, 2001.

[KST11] A. Kulik, H. Shachnai, T. Tamir. Approximations for Monotone and Non-monotone Submodular Maximization with Knapsack Constraints. arXiv:1101.2940v1, 2011.

[KST09] A. Kulik, H. Shachnai and T. Tamir. Maximizing submodular set functions subject to multiple linear constraints. Proc. of ACM-SIAM SODA, 545-554, 2009.

[LMNS09] J. Lee, V. Mirrokni, V. Nagarajan, and M. Sviridenko. Maximizing non-monotone submodular functions under matroid and knapsack constraints. In Proceedings of the 41st Annual ACM Symposium on Theory of Computing (STOC), 2009. 
[OV11] S. Oveis Gharan and J. Vondrák. Submodular maximization by simulated annealing. In Proc. of the 22nd Annual ACM/SIGACT-SIAM Symposium on Discrete Algorithms (SODA), 2011.

[S04] M. Sviridenko. A note on maximizing a submodular set function subject to knapsack constraint. Operations Research Letters 32, 41-43, 2004.

[Sch03] A. Schrijver. Combinatorial optimization - polyhedra and efficiency. Springer, 2003.

[SU07] A. Schulz, and N. Uhan. Encouraging cooperation in sharing supermodular costs Approximation, Randomization, and Combinatorial Optimization. Algorithms and Techniques, 271-285, 2007.

[V08] J. Vondrák. Optimal approximation for the submodular welfare problem in the value oracle model. In STOC, pages 67-74, 2008.

[V09] J. Vondrák. Symmetry and approximability of submodular maximization problems. In Proc. of IEEE FOCS, 251-270, 2009.

[V10] J. Vondrák. A note on concentration of submodular functions. Manuscript, 2010. 